\title{
Access to Formal Maternal Health Services and Informal Supports for Resettled Refugee Mothers in the Postnatal Period: A Scoping Review Protocol
}

\section{Emma Stirling Cameron ( $\nabla$ emma.cameron@dal.ca )}

Dalhousie University https://orcid.org/0000-0001-9388-2556

\section{Benjamin DuPlessis}

Dalhousie University Faculty of Medicine

\section{Megan Aston}

Dalhousie University School of Nursing

Howard Ramos

University of Western Ontario: Western University

\section{Lois Jackson}

Dalhousie University

\section{Protocol}

Keywords: Refugees, postnatal health, social support, maternal health, women, scoping review

Posted Date: November 5th, 2020

DOl: https://doi.org/10.21203/rs.3.rs-101217/v1

License: (9) This work is licensed under a Creative Commons Attribution 4.0 International License. Read Full License 


\section{Abstract}

Background: The postnatal period is a critical time when women may need access to formal maternal health services (e.g., family physicians) and informal supports (e.g., family, friends). Resettled refugee women often encounter a myriad of barriers and challenges when attempting to access these services and supports in resettlement countries, including language and communication differences, socioeconomic constraints, challenges navigating foreign healthcare systems, and reduced social support networks. This has contributed to health inequities among postnatal resettled refugee women, including low self-reported health, five-times higher rates of postpartum depression, and social isolation. The purpose of this review is to understand access to formal maternal health services and informal supports for resettled refugee women during the postnatal period.

Methods: This review will be conducted in accordance with JBI methodology for scoping reviews. A comprehensive search strategy, developed with a librarian scientist, will be used to identify relevant sources. Titles, abstracts, and full texts will be evaluated against inclusion criteria. Evidence from peerreviewed and gray literature published after 2003 will be included. This review will focus only on studies conducted in middle- and high-income resettlement countries. Information will be extracted by two independent reviewers using a screening tool. Data will be synthesized and presented narratively, with tables and figures where appropriate.

Discussion: This scoping review will provide an overview of resettled refugee women's experiences during the postnatal period, with a focus on understanding access to health services and the availability and use of informal supports. It is expected that this review will outline both the barriers and facilitators affecting resettled refugee women seeking formal and informal care during the postnatal period. Findings will help to inform policy direction and future interventions seeking to improve postnatal care for resettled refugee women.

Systematic Review Registration: This scoping review protocol has been registered with the Open Science Framework database, https://osf.io/rkqj6.

\section{Background}

\section{Global migration crisis}

The global refugee crisis has reached record proportions over the past decade, with the number of refugees doubling since $2012 .{ }^{1}$ An unprecedented 70.8 million people are currently displaced across the world-25.9 million of whom are refugees. A refugee is defined as someone who has been forced to flee their country of origin due to conflict, persecution, or violence, and are seeking refuge in another country. ${ }^{1}$ In 2018 alone, 13.6 million people were newly displaced as a result of conflict or persecution. ${ }^{1}$ International resettlement remains a life-saving tool for many vulnerable refugees who are unable to return to their home countries. In 2018, 92,400 refugees were resettled in 25 predominantly high-income 
countries, including Canada (28,100), the United States (22,900), and Australia $(12,700) .{ }^{1}$ A large proportion of resettled refugees were women and girls, many of whom already have children or are of childbearing age. ${ }^{2}$ The experiences of forced migration and resettlement have far-reaching impacts on the health of refugee women, particularly during the postnatal period. ${ }^{3}$

\section{The importance of formal health services and informal supports during the postnatal period}

The postnatal period is often an exciting time for new parents. It is also a critical period when mothers may need access to health services and informal supports to treat a variety of psychosocial and physical health concerns. ${ }^{4}$ Nearly $95 \%$ of women report experiencing one or more maternal health concerns (i.e., relating to the health of the mother) within the first six months of childbirth, including mastitis, prolonged vaginal bleeding, urinary incontinence, vaginal or caesarian site pain, postpartum blues or depression, and social isolation. ${ }^{4}$ Untreated health problems are directly related to a decrease in women's emotional wellbeing and functional abilities, including an inability to complete routine household activities, provide childcare, and return to work. ${ }^{5}$ Timely and effective postpartum healthcare provided by formal healthcare professionals (e.g., family physicians, obstetricians, psychologists, doulas) can prevent short- and longterm consequences of unrecognised and poorly managed health morbidities. ${ }^{6}$

In addition to the availability of formal health services, informal supports provided by spouses, relatives, friends or associates, plays a crucial role in the health and wellbeing of new mothers. ${ }^{7-9}$ The World Health Organization states that access to informal support persons is necessary for maternal and infant wellbeing and contributes to a positive transition into motherhood. ${ }^{10}$ Informal supports may be particularly useful among low-income families who experience financial barriers to health services, particularly in countries who do not provide universal healthcare (e.g., the United States). ${ }^{11}$ Women with low levels of informal support are more likely to report having postpartum depression and a lower quality of life, when compared to women who report feeling well supported. ${ }^{9}$ Persistent postpartum depression has been correlated with insecure infant attachment, and lower infant cognitive and psychomotor development. ${ }^{12}$ Social support acts as a protective factor against depressive symptoms, enhances maternal parental self-efficacy, and improves coping during the postnatal period. 7,13

Evidence suggests that access to both formal health services and informal support during the postnatal period are critical to supporting maternal health. Yet access to services and supports remains a challenge for many resettled refugee women across the globe.

\section{Barriers to formal maternal health services and informal supports among resettled refugee women}

Resettled refugee women report experiencing a myriad of barriers when attempting to or actually accessing healthcare services, often as a result of the complex reality resettled refugee women experience. ${ }^{14}$ Women are required to cope with the challenges of new parenthood in conjunction with the financial and sociocultural stressors of resettlement. ${ }^{15}$ Women report prioritizing the needs of their family (e.g., accessing affordable housing, caring for other children) over their own personal health. ${ }^{3,14}$ 
Language and communication differences are commonly reported barriers, which can influence women's abilities to convey their concerns and interpret healthcare providers' instructions and advice. ${ }^{15,16}$ While interpretation services are sometimes provided, there are issues of confidentiality, and appointments may not be long enough to accommodate interpreters. ${ }^{16}$ Financial constraints-including a lack of health insurance, cost of services or prescription medications, and a limited access to childcare and transportation-can deter women from engaging with the healthcare system. ${ }^{3,15,16}$ Women further report feeling confused navigating the health system of their host country, not knowing that supports exist for particular concerns-especially in the treatment of postpartum depression. ${ }^{13}$ Perceptions of racism, discrimination, and culturally insensitive care can negatively influence women's trust and future engagement with the healthcare system. ${ }^{3,15}$

It is not uncommon for resettled refugee women to have lost or been separated from family or friends during their time spent as a refugee and throughout the resettlement process. ${ }^{13}$ Many refugee women have reduced social networks upon arriving in their host country, resulting in lower levels of available informal supports during the postnatal period. ${ }^{17}$ Linguistic and cultural barriers often impede resettled refugee women's ability to socially integrate into their host country. Resettled refugee women with social networks containing fewer women, relatives, and people from their own ethic background are more likely to experience high rates of depressive symptoms. ${ }^{18}$ Women have reported feeling overwhelmed and frightened about having to care for a new baby without the advice and support of their family. ${ }^{19}$ No informal supports to help with childcare has been connected to delayed or missed medical appointments. ${ }^{17}$

\section{Postnatal health challenges among resettled refugee women}

Reduced access to relevant postnatal health services and a reduced informal support network has the ability to negatively impact the health of resettled refugee women. Refugee women are significantly more likely to have a poorer postpartum health status ${ }^{20}$ and a greater number of unmet physical and mental health concerns when compared to Canadian-born women. ${ }^{21}$ Resettled refugee women in Sweden had higher risk of poor self-rated health compared to Swedish-born women. ${ }^{22}$ Rates of postpartum depression are five-times higher among refugee women when compared to Canadian-born women. ${ }^{23}$ Refugee women underutilise healthcare services, ${ }^{24}$ and rate community health services unfavourably. ${ }^{20}$ The accessibility of formal healthcare services and the availability of informal supports is crucial for supporting refugee mothers during the postnatal period.

\section{Methods/design}

This scoping review seeks to addressing the following: What evidence is currently available to describes access to formal maternal health services and informal supports for resettled refugee women during the postnatal period? 
The proposed scoping review will be conducted in accordance with the Joanna Briggs Institute methodology for scoping reviews. ${ }^{25}$ The population-concept-context (PCC) framework will be used to align the research question with study selection. ${ }^{25}$ See Additional File 1 for PRISMA-P flowchart.

\section{Participants}

This review will consider studies that focus on refugee women who have been resettled in designated UNHCR resettlement countries. Immigrant, migrant, and refugee-claimant women will not be included. No restrictions will be placed on country of origin, women's age, religion, sexual orientation, or marital status. Studies that have elicited healthcare providers' perceptions on access to postnatal services and supports for refugee women will also be included.

\section{Concepts}

Access to postnatal health services. For the purposes of this study, the postnatal period will be defined as the first 12 months after childbirth. Maternal, not infant health, will be the focus of this review. Formal health services will include any health services provided by a healthcare professional. This will include, but is not limited to, services provided by family physicians, obstetricians and gynecologists, nurses, pharmacists, social workers, psychologists, counsellors, physiotherapists, massage therapists, doulas, midwives. Access to healthcare has been defined as "the opportunity to reach and obtain appropriate healthcare services in situations of perceived need for care" (p.4). ${ }^{26}$ Access to healthcare has five key features. This includes the possibility of the user to: 1) identify their healthcare needs, 2) seek out healthcare services, 3) reach necessary healthcare resources, 4) obtain or use healthcare services, and 5) be offered services appropriate to the needs of care. ${ }^{26}$

Informal supports. Informal supports can include: informational support (providing advice or guidance), instrumental support (offering material aid or assistance with tasks), and emotional support (acting as a confident, bolstering self-esteem) during the postnatal period, as provided by spouses, relatives, friends or associates (in-person and online). ${ }^{8}$

\section{Context}

This review will consider studies have been conducted in UNHCR resettlement countries. The complete list of countries that have historically resettled refugees (between the years 2003 and 2020) in collaboration with the UNHCR have been listed in Table $1 .{ }^{27}$ This includes predominately high and uppermiddle income countries. ${ }^{28}$ Access to services in refugee camps or other temporary settlements will not be included in this review. Refugees of any ethnicity or country of origin will be included.

\section{Types of sources}

This scoping review will consider all study designs including randomized controlled trials, nonrandomized controlled trials, before and after studies and interrupted time-series studies. In addition, 
analytical observational studies including, prospective and retrospective cohort studies, case-control studies and analytical cross-sectional studies, will be considered for inclusion. This review will also consider descriptive observational study designs including case series, individual case reports, and descriptive cross-sectional studies.

Qualitative studies that focus on qualitative data including, but not limited to, designs such as phenomenology, grounded theory, ethnography, qualitative description, action research and feminist research will also be considered. Other sources that meet inclusion criteria, such as systematic reviews, dissertations, gray literature, opinion papers, and research papers that include sub-analyses, will be considered for inclusion. Studies published in English will be included. Only studies published after 2003 will be included, as that was when UNHCR resettlement data was made publicly available.

\section{Search strategy}

The JBI three-step search strategy will be implemented in this review. ${ }^{25}$ The search strategy, developed in conjunction with a librarian scientist, will aim to locate both published and unpublished primary studies. An initial limited search of CINAHL (Appendix I) was undertaken to identify articles on the topic. The text words contained in the titles and abstracts of relevant articles, and the index terms used to describe the articles were used to develop a full search strategy for CINAHL. The search strategy, including all identified keywords and index terms will be adapted for each included information source. The reference lists of articles selected for full text review will be screened for additional studies.

\section{Information sources}

The databases to be searched include CINAHL (EBSCO), MEDLINE (OVID), Embase (Elsevier), Studies on Women \& Gender Abstracts (Taylor and Francis), Academic Search Premier (EBSCO), Sociological Abstracts (ProQuest), Social Services Abstracts (ProQuest), PAIS Index (ProQuest), Public Affairs Index (EBSCO), and PsycINFO (American Psychological Association). Sources of unpublished studies and gray literature to be searched include: The United Nations, the United Nations High Commissioner on Refugees, the International Organization for Migration, Centre for Disease Control, United Nations Population Fund, the World Health Organization, Google Scholar, ProQuest Dissertations and Theses Databases, Migration Policy Institute, Refugee Council, Canadian Council for Refugees, Gray Literature Report [via New York Academy of Medicine website],

\section{Study selection}

Following the search, all identified records will be collated and uploaded into Covidence (Veritas Health Innovation, Melbourne, Australia) and duplicates removed. Titles and abstracts will then be screened by two independent reviewers for assessment against the inclusion criteria for the review. Potentially relevant papers will be retrieved in full and their citation details imported into the Joanna Briggs Institute's System for the Unified Management, Assessment and Review of Information (JBI SUMARI) (The Joanna Briggs Institute, Adelaide, Australia). The full text of selected citations will be assessed in detail against 
the inclusion criteria by two independent reviewers. Reasons for exclusion of full text papers that do not meet the inclusion criteria will be recorded and reported in the scoping review. Any disagreements that arise between the reviewers at each stage of the selection process will be resolved through discussion or with a third reviewer. The results of the search will be reported in full in the final scoping review and presented in a Preferred Reporting Items for Systematic Reviews and Meta-analyses Extension for Scoping Reviews (PRISMA-ScR) flow diagram. ${ }^{29}$

\section{Data extraction}

Data will be extracted from papers included in the scoping review by two independent reviewers using a data extraction tool developed by the reviewers. The data extracted will include specific details about the population, concept, context, methods, and key findings relevant to the review question. A draft data extraction tool is provided in Appendix II. This tool has been piloted by ESC. The draft data extraction tool may be modified and revised as necessary during the process of extracting data from each included paper. Modifications will be detailed in the full scoping review. Any disagreements that arise between the reviewers will be resolved through discussion, or with a third reviewer. Authors of papers will be contacted to request missing or additional data, where required.

\section{Data presentation}

The extracted data will be presented in diagrammatic or tabular form in a manner that aligns with the objective of this scoping review. A narrative summary will accompany the tabulated and/or charted results and will describe how the results relate to the reviews objective and question.

\section{Discussion}

This scoping review will identify, collect, and synthetize available literature on refugee women accessing formal services and informal supports during the postnatal period in resettlement countries. A preliminary search of PROSPERO, MEDLINE, Open Science Frameworks, and the Joanna Briggs Institute Database of Systematic Reviews and Implementation Reports was conducted and no current or underway scoping reviews or systematic reviews on the topic were identified. There have been several scoping and systematic reviews on the perinatal health outcomes and experiences of refugee, immigrant, and asylumseeking women. ${ }^{3,15,30}$ None of these reviews have examined the postpartum period in isolation and have not isolated the experiences of refugee women, who often face unique challenges when compared to immigrants. ${ }^{15,30}$ Refugee women are more likely to experience socioeconomic barriers, experience disruptions in family life, and have a history of trauma or reduced access to services. In addition, the provision of informal supports has largely been excluded from other reviews. Previous literature has shown that informal supports, including providing childcare, cooking meals, offering advice and emotional support, can prove invaluable for new mothers in the postpartum period and can mitigate against the development of postpartum depression. ${ }^{8,18}$ This review will look at both access to formal health services and informal supports among postnatal refugee women in resettlement countries. 
The research team will utilise information learned in this review to inform a project seeking to understand access to formal health services and informal supports among postnatal Syrian refugee women in Canada. Concepts and topics uncovered in the review will be integrated into the development of the guide for this project and may help to inform the development of a local best practice guide on providing postnatal care for resettled refugees. This review will shed light on the experiences of resettled refugee women during the postnatal period. Critically, this review will highlight barriers and facilitators to health services and informal supports among postnatal refugee women in resettlement countries. By collecting findings of barriers to accessing services and supports for refugee women, we can inform further scholarship into the access issues facing refugee women; we can also begin discussions around targeted interventions and policy to create more equitable access to care and support in resettlement countries. Based on preliminary work, we anticipate potential limitations due to differential healthcare systems among resettlement countries, which may limit the generalizability of the results.

\section{Abbreviations}

UNHCR: United Nations High Commissioner for Refugees

WHO: World Health Organization

PCC: Population-concept-context

PRISMA-P: Preferred reporting items for systematic review and meta-analysis protocols

IRCC: Immigration, refugees and citizenship Canada

\section{Declarations}

\section{Ethics approval and consent to participate.}

None required. Not applicable.

\section{Consent for publication.}

Not applicable.

\section{Availability of data and materials.}

All data is available from public sites and databases.

\section{Competing interests.}

None.

\section{Funding.}


The first author (ESC) has received student support funding from the following sources: The Faculty of Graduate Studies at Dalhousie University, the IWK Health Centre, BRIC NS, and the Social Sciences and Humanities Research Council of Canada.

\section{Authors' contributions.}

E. Stirling Cameron is the first author and one of two reviewers for the proposed review. She worked closely with the librarian scientist to develop and execute the search strategy and drafted the scoping review protocol paper. B. DuPlessis is the second reviewer for the proposed review and has contributed to the writing and editing of the protocol. He is an upper-year medical student with significant experience working with refugee families in clinical and non-profit settings. M. Aston is a professor in nursing with extensive knowledge in the field of postoperative care and service delivery and is well versed in JBI methodology. She has given guidance and feedback on the protocol and will continue to ensure JBI methods are upheld and will assist with conflict resolution. H. Ramos is a professor in sociology who specializes in immigration and refugee research. He provided feedback on the protocol and will continue to provide advice and edits on future drafts. L. Jackson is the supervising author. She has worked closely with E. Stirling Cameron on developing the research question, protocol, and search strategy. She has and will continue to provide extensive feedback. All authors have read and provided edits on this protocol.

\section{Acknowledgements.}

The authors would like to acknowledge Shelley McKibbon* for her assistance in developing the search strategy for this review.

*Health and Human Performance Librarian, Kellogg Health Sciences Library, Dalhousie University, Carleton Campus.

\section{References}

1. UNHCR. Global trends: Forced displacement in 2018 [Internet]. 2019. Available from: https://www.unhcr.org/globaltrends2018/

2. IRCC. Syrian refugees family composition. 2016.

3. Heslehurst N, Brown H, Pemu A, Coleman H, Rankin J. Perinatal health outcomes and care among asylum seekers and refugees: A systematic review of systematic reviews. BMC Med. 2018;16(1):125.

4. Brown S, Lumley J. Maternal health after childbirth: Results of an australian population based survey. Br J Obstet Gynaecol. 1998;105(2):156-61.

5. Webb DA, Bloch JR, Coyne JC, Chung EK, Bennett IM, Culhane JF. Postpartum physical symptoms in new mothers: Their relationship to functional limitations and emotional well-being. Birth. 2008;35(3):179-87. 
6. Haran C, van Driel M, Mitchell BL, Brodribb WE. Clinical guidelines for postpartum women and infants in primary care-a systematic review. BMC Pregnancy Childbirth. 2014;14(1):1-9.

7. Emmanuel E, St John W, Sun J. Relationship between social support and quality of life in childbearing women during the perinatal period. JOGNN - J Obstet Gynecol Neonatal Nurs [Internet]. 2012 Nov 1 [cited 2020 May 16];41(6):E62-70. Available from: https://linkinghub.elsevier.com/retrieve/pii/S0884217515312296

8. Eastwood JG, Jalaludin BB, Kemp LA, Phung HN, Barnett BE. Relationship of postnatal depressive symptoms to infant temperament, maternal expectations, social support and other potential risk factors: Findings from a large Australian cross-sectional study. BMC Pregnancy Childbirth [Internet]. 2012 [cited 2020 May 16];12(148):1-11. Available from: http://www.biomedcentral.com/14712393/12/148

9. Webster J, Nicholas C, Velacott C, Cridland N, Fawcett L. Quality of life and depression following childbirth: Impact of social support. Midwifery [Internet]. 2011;27(5):745-9. Available from: http://dx.doi.org/10.1016/j.midw.2010.05.014

10. WHO. The World Health Report: Make every mother and child count [Internet]. World Health Report. 2005. Available from: https://www.who.int/whr/2005/whr2005_en.pdf?ua=1

11. Radey M. Informal support among low-income mothers post welfare reform: A systematic review. Vol. 27, Journal of Child and Family Studies. 2018. p. 3782-805.

12. Cornish AM, McMahon CA, Ungerer JA, Barnett B, Kowalenko N, Tennant C. Postnatal depression and infant cognitive and motor development in the second postnatal year: The impact of depression chronicity and infant gender. Infant Behav Dev. 2005;28(4):407-17.

13. Higginbottom GMA, Hadziabdic E, Yohani S, Paton P. Immigrant women's experience of maternity services in Canada: A meta-ethnography. Midwifery [Internet]. 2014;30(5):544-59. Available from: http://dx.doi.org/10.1016/j.midw.2013.06.004

14. Peláez S, Hendricks KN, Merry LA, Gagnon AJ. Challenges newly-arrived migrant women in Montreal face when needing maternity care: Health care professionals' perspectives. Global Health [Internet]. 2017 [cited 2019 Sep 14];13(1). Available from: https://globalizationandhealth-biomedcentralcom.ezproxy.library.dal.ca/track/pdf/10.1186/s12992-016-0229-x

15. Khanlou N, Haque N, Skinner A, Mantini A, Kurtz Landy C. Scoping review on maternal health among immigrant and refugee women in Canada: Prenatal, intrapartum, and postnatal care. J Pregnancy. 2017;2017(1):1-14.

16. Riggs E, Davis E, Gibbs L, Block K, Szwarc J, Casey S, et al. Accessing maternal and child health services in Melbourne, Australia: Reflections from refugee families and service providers. BMC Health Serv Res. 2012;12(1):1-16.

17. Higginbottom GM, Safipour J, Yohani S, O’Brien B, Mumtaz Z, Paton P, et al. An ethnographic investigation of the maternity healthcare experience of immigrants in rural and urban Alberta, Canada. BMC Pregnancy Childbirth. 2016;16(1):1-15. 
18. Zelkowitz P, Schinazi J, Katofsky L, Saucier JF, Valenzuela M, Westreich R, et al. Factors associated with depression in pregnant immigrant women. Transcult Psychiatry. 2004;41(4):445-64.

19. Ahmed A, Stewart DE, Teng L, Wahoush O, Gagnon AJ. Experiences of immigrant new mothers with symptoms of depression. Arch Womens Ment Health. 2008;11(4):295-303.

20. Ganann R, Sword W, Black M, Carpio B. Influence of maternal birthplace on postpartum health and health services use. J Immigr Minor Heal. 2012;14(2):223-9.

21. Gagnon AJ, Dougherty G, Wahoush O, Saucier JF, Dennis CL, Stanger E, et al. International migration to Canada: The post-birth health of mothers and infants by immigration class. Soc Sci Med. 2013;76(1):197-207.

22. Liu C, Ahlberg M, Hjern A, Stephansson O. Perinatal health of refugee and asylum-seeking women in Sweden 2014-17: A register-based cohort study. Eur J Public Health. 2019;29(6):1048-55.

23. Stewart DE, Gagnon A, Saucier JF, Wahoush O, Dougherty G. Postpartum depression symptoms in newcomers. Can J Psychiatry. 2008;53(2):121-4.

24. Chan M, Johnston $C$, Bever A. Exploring health service underutilization: A process evaluation of the newcomer women's health clinic. J Immigr Minor Heal. 2018;20(4):920-5.

25. Peters MDJ, Godfrey C, Mclnerney P, Baldini Soares C, Khalil H, Parker D. Chapter 11: Scoping Reviews. In: Aromataris E, Munn Z, editors. Joanna Briggs Institute Reviewer's Manual. 2017.

26. Levesque J-F, Harris MF, Russell G. Patient-centred access to health care: conceptualising access at the interface of health systems and populations. Int J Equity Health. 2013;12(18):1-9.

27. UNHCR. Resettlement data finder [Internet]. 2020 [cited 2020 May 17]. Available from: https://rsq.unhcr.org/en/\#2R7g

28. The World Bank. World Bank country and lending groups [Internet]. 2019. Available from: https://datahelpdesk.worldbank.org/knowledgebase/articles/906519-world-bank-country-andlending-groups

29. Tricco AC, Lillie E, Zarin W, O’Brien KK, Colquhoun H, Levac D, et al. PRISMA extension for scoping reviews (PRISMA-ScR): Checklist and explanation. Ann Intern Med. 2018;169(7):467-73.

30. Anderson FM, Hatch SL, Comacchio C, Howard LM. Prevalence and risk of mental disorders in the perinatal period among migrant women: A systematic review and meta-analysis. Arch Womens Ment Health. 2017;20(3):449-62.

\section{Table}


Table 1

Resettlement countries listed by the UNHCR (2003-2020) ${ }^{27}$

\begin{tabular}{|ll|}
\hline Argentina & Latvia \\
\hline Australia & Liechtenstein \\
\hline Austria & Lithuania \\
\hline Belarus & Luxembourg \\
\hline Belgium & Malta \\
\hline Brazil & Mexico \\
\hline Bulgaria & Monaco \\
\hline Canada & The Netherlands \\
\hline Chile & New Zealand \\
\hline Croatia & Norway \\
\hline Cyprus & Paraguay \\
\hline Czechia & Poland \\
\hline Denmark & Portugal \\
\hline Estonia & Romania \\
\hline Finland & Slovenia \\
\hline France & Spain \\
\hline Germany & Sweden \\
\hline Hungary & Switzerland \\
\hline Iceland & United Kingdom \\
\hline Ireland & United States of America \\
\hline Italy & Uruguay \\
\hline Japan & \\
\hline
\end{tabular}

\section{Appendix}

\section{Appendix l: Search strategy}

CINAHL (EBSCO): Searched June $2^{\text {nd }}, 2020$. 


\begin{tabular}{|c|c|c|}
\hline Search & Query & $\begin{array}{l}\text { Records } \\
\text { retrieved }\end{array}$ \\
\hline 1 & woman OR women OR female OR mothers & $2,235,761$ \\
\hline 2 & $\begin{array}{l}\text { refugee* OR ( ((displaced OR resettled) W1 (person* OR people OR women OR } \\
\text { woman OR population*)) ) OR newcomer* }\end{array}$ & 9,862 \\
\hline 3 & (MH "Women+") & 34,270 \\
\hline 4 & (MH "Refugees") & 7,627 \\
\hline 5 & $\begin{array}{l}\text { Argentina OR Australia OR Austria OR Belarus OR Belgium OR Brazil OR } \\
\text { Bulgaria OR Canada OR Chile OR Croatia OR Cyprus OR Czechia OR Denmark } \\
\text { OR Estonia OR Finland OR France OR Germany OR Hungary OR Iceland OR } \\
\text { Ireland OR Italy OR Japan OR Latvia OR Liechtenstein OR Lithuania OR } \\
\text { Luxembourg OR Malta OR Mexico OR Monaco OR The Netherlands OR New } \\
\text { Zealand OR Norway OR Paraguay OR Poland OR Portugal OR Romania OR } \\
\text { Slovenia OR Spain OR Sweden OR Switzerland OR United Kingdom OR United } \\
\text { States of America OR Uruguay }\end{array}$ & 923,551 \\
\hline 6 & $\begin{array}{l}\text { (MH "Argentina") OR (MH "Australia+") OR (MH "Austria") OR (MH "Byelarus") } \\
\text { OR (MH "Belgium") OR (MH "Brazi") OR (MH "Bulgaria") OR (MH "Canada+") } \\
\text { OR (MH "Chile") OR (MH "Croatia") OR (MH "Mediterranean Islands") OR (MH } \\
\text { "Czech Republic") OR (MH "Denmark") OR (MH "Estonia") OR (MH "Finland") } \\
\text { OR (MH "Norway") OR (MH "Sweden") OR (MH "France") OR (MH "Germany+") } \\
\text { OR (MH "Iceland") OR (MH "Ireland") OR (MH "Italy") OR (MH "Liechtenstein") } \\
\text { OR (MH "Luxembourg") OR (MH "Netherlands") OR (MH "Switzerland") OR (MH } \\
\text { "United Kingdom+") OR (MH "Spain") OR (MH "Portugal") OR (MH "Monaco") } \\
\text { OR (MH "Poland") OR (MH "Slovenia") OR (MH "Romania") OR (MH "Japan") } \\
\text { OR (MH "Mexico") OR (MH "United States+") OR (MH "New Zealand") OR (MH } \\
\text { "Uruguay") OR (MH "Paraguay") OR (MH "Latvia") OR (MH "Lithuania") OR (MH } \\
\text { "Hungary") }\end{array}$ & $1,669,562$ \\
\hline 7 & $\begin{array}{l}\text { (MH "Postnatal Period") OR (MH "Lactation") OR (MH "Puerperium") OR (MH } \\
\text { "Postnatal Care") OR (MH "Lactation Suppression") OR (MH "Puerperal } \\
\text { Disorders+") OR (MH "Maternal Health Services") OR (MH "Maternal Welfare") } \\
\text { OR (MH "Maternal-Child Health") }\end{array}$ & 43,215 \\
\hline 8 & $\begin{array}{l}\text { Postpartum OR postnatal OR “post-partum" OR “post natal” OR ((new OR } \\
\text { nursing OR newborn* OR infant* OR neonate*) N2 (mother* OR parent OR } \\
\text { parents)) }\end{array}$ & 134,173 \\
\hline 9 & $\begin{array}{l}\text { (MH "Midwives+") OR (MH "Nurse Midwives") OR (MH "Lay Midwives") OR } \\
\text { (MH "Nurse-Midwifery Service") OR (MH "Midwifery Service") OR (MH } \\
\text { "Doulas") OR (MH "Obstetrics") OR (MH "Gynecology") OR (MH "Diagnosis, } \\
\text { Obstetric+") OR (MH "Obstetric Service") OR (MH "Obstetric Patients") OR (MH } \\
\text { "OB-GYN Nurse Practitioners") OR (MH "Gynecologic Nursing") OR (MH } \\
\text { "Gynecologic Care") OR (MH "Gynecologic Examination") OR (MH "Diagnosis, } \\
\text { Gynecologic+") OR (MH "Diagnosis, Ob-Gyn+") OR (MH "Surgery, } \\
\text { Gynecologic+") }\end{array}$ & 81,601 \\
\hline 10 & obstetric* OR gynecolog* OR "OB GYN" OR "OBGYN" & 73,653 \\
\hline 11 & $\begin{array}{l}\text { (MH "Support, Psychosocial+") OR (MH "Social Isolation") OR (MH "Social } \\
\text { Inclusion") OR (MH "Social Participation") OR (MH "Spirituality") OR (MH } \\
\text { "Psychosocial Deprivation") OR (MH "Intergenerational Relations") OR (MH } \\
\text { "Family Conflict") OR (MH "Friendship") OR (MH "Adult-Child Relations") OR } \\
\text { (MH "Patient-Family Relations") OR (MH "Nurturing Behavior") OR (MH }\end{array}$ & 409,293 \\
\hline
\end{tabular}


"Professional-Family Relations") OR (MH "Nurse-Patient Relations") OR (MH

"Physician-Patient Relations") OR (MH "Caring") OR (MH "Dissent and

Disputes") OR (MH "Family Functioning+") OR (MH "Family Relations") OR

(MH "Maternal Behavior") OR (MH "Parent-Child Relations+") OR (MH "ParentInfant Relations+") OR (MH "Parenting") OR (MH "Paternal Behavior") OR (MH "Family Separation") OR (MH "Sibling Relations") OR (MH "Spouses") OR (MH

"Family+")

12 Social OR socially OR support* OR family OR parent OR mother OR father OR friends OR community OR neighbo* OR psychosocial OR informal OR network

\begin{tabular}{|lll|}
\hline 13 & S1 OR S3 & $2,236,217$ \\
\hline 14 & S2 OR S4 & 9,862 \\
\hline 15 & S5 OR S6 & $1,774,757$ \\
\hline 16 & S7 OR S8 & 148,682 \\
\hline 17 & S9 or S10 OR S11 OR S12 & $1,902,456$ \\
\hline 18 & S13 AND S14 AND S15 AND S16 AND S17 & 223 \\
\hline
\end{tabular}

Appendix II: Data extraction instrument 


\begin{tabular}{|l|}
\hline Scoping Review Details \\
\hline Title \\
\hline Year \\
\hline Review objective/s \\
\hline Review question/s \\
\hline Inclusion/Exclusion Criteria \\
\hline Population \\
\hline Major Concept \\
\hline Context \\
\hline Type of Study \\
\hline Study Details and Characteristics \\
\hline Study citation details (e.g. author/s, date, title, journal, volume, issue, pages) \\
\hline Host country \\
\hline Postnatal services/supports examined \\
\hline Participants (details e.g. age, second-time parent, country of origin, number) \\
\hline Details/Results extracted from study (in relation to the concept of the scoping review) \\
\hline Barriers to services/supports \\
\hline Facilitators to services/supports \\
\hline Other key findings \\
\hline Implications on health (if stated) \\
\hline Gaps identified \\
\hline Recommendations \\
\hline Additional notes \\
\hline
\end{tabular}

\section{Supplementary Files}

This is a list of supplementary files associated with this preprint. Click to download.

- PRISMAPchecklist.docx 